\title{
Hidden Multiverse Extraterrestrial Super Civilizations
}

\author{
Alexander Alexandrovich Antonov \\ Research Centre of information Technologies “TELAN Electronics”, Kiev, Ukraine \\ Email: telan@bk.ru
}

How to cite this paper: Antonov, A.A. (2017) Hidden Multiverse Extraterrestrial Super Civilizations. Natural Science, 9, 4362.

https://doi.org/10.4236/ns.2017.93005

Received: February 15, 2017

Accepted: March 28, 2017

Published: March 31, 2017

Copyright $\odot 2017$ by author and Scientific Research Publishing Inc. This work is licensed under the Creative Commons Attribution International License (CC BY 4.0).

http://creativecommons.org/licenses/by/4.0/

\begin{abstract}
Theoretical and experimental evidence of physical reality of imaginary numbers using interpretation of Ohm's law for electrical AC circuits proposed in 1897 by Steinmetz was presented. Other pieces of evidence are mentioned as well. The use of the general scientific principle of the physical reality of imaginary numbers allowed to prove that the relativistic formulas in the existing version of the special relativity theory are wrong, as well as to deny the principle of non-exceeding the speed of light and the hypothesis of monoverse. Corrected relativistic formulas are given and on their basis a theory of hidden Multiverse is proposed, parallel universes of which are mutually invisible. Using data obtained by the spacecrafts WMAP and Planck, it is proved that the hidden Multiverse has a quaternion structure, exists in the six-dimensional space, and forms Hyperverse together with other Multiverses. It is shown that the parallel universes of the hidden Multiverse are continuously drifting in multidimensional space, touch each other in many places and are even partially immersed in each other, forming portals. It is alleged that the search for extraterrestrial life in deep space is devoid of meaning, as you can even walk to the adjacent parallel universes through portals, which are anomalous zones on Earth. Moreover, extraterrestrial super civilizations have already found us long time ago and are located on Earth. The attempts to contact these super civilizations are meaningless as well, because such contacts are possible only at their wish. Consequently, to make the contacts possible, we need to convince super civilizations that human civilization is of interest to them and that it has a sufficiently high intelligence. It is shown how the human super intelligence over can be realized.
\end{abstract}

\section{Keywords}

Imaginary Numbers, Special Relativity Theory, Dark Matter, Dark Energy, Multiverse, Hyperverse, Portals, Civilization, Intelligence, Technological Singularity 


\section{Introduction}

Established at the beginning of the last century by Joseph Larmor [1], the Nobel Prize winner Hendrik Antoon Lorentz [2], Jules Henri Poincaré [3], the winner of the Nobel Prize Albert Einstein [4] and other eminent scientists, special relativity theory (SRT) [5] [6] [7] is still flawed despite all of its universally recognized merits. Its second postulate [8] undergoes especially a lot of criticism, having in addition to offered original formulation [4] in the form of principle of the constant velocity of light, the other two supposedly identical to or following from it (that was not proved anywhere) formulations in the form of the principle of non-exceeding the speed of light and approval of the physical unreality of the imaginary numbers.

Since modern physics is largely founded not on experimentally obtained data, but on those or other postulates, the corresponding theories can be incontrovertibly refuted only by means of experiments. And so in the early twenty-first century attempts of refutation of the principle of non-exceeding the speed of light in SRT were made by collaboration of MINOS [9] on the US Tevatron collider and collaboration of OPERA [10] on the European Large Hadron Collider. However, MINOS experiment has been ignored by the physics community as not sufficiently reliable, and OPERA experiment has been refuted in six months by ICARUS experiment [11]. What is more, during those six months several dozen scientific publications have been devoted to the search for OPERA experiment refutation options. Some of them even claimed that, since the principle of non-exceeding the speed of light is fundamental in SRT, then, if OPERA experiment had proved successful, its authors would have deserved the Nobel Prize.

However, as noted by the author of the concept of 'open society' Karl Raimund Popper [12]: "...the struggle of opinions in scientific theories is inevitable and is a necessary prerequisite for the development of science". Therefore, it turned out that at the same time the challenge of physical reality of imaginary numbers [13] [14] [15] [16] [17] was successfully solved, but using different experiments, from which, as shown below, follows the fallacy of the principle of non-exceeding the speed of light. However, oddly enough, for this event, in contrast to OPERA experiment, the physics community has shown no interest over the past few years and the Nobel Prize had not been recalled.

This circumstance unwittingly begs the simple explanation that the physical community did not need scientific truth, but the support of an erroneous public opinion about the alleged infallibility of SRT. And as such, long-term unique OPERA experiment, which has failed to refute the principle of non-exceeding the speed of light appeared as the most opportune and absolutely reliable alternative experiments described below, managed to still prove the fallacy of SRT, were, on the contrary, very inappropriate.

\section{Why Is the Principle of Non-Exceedance the Speed of Light Is Fundamental in SRT}

Why is the principle of non-exceeding the speed of light was so necessary in 
SRT? Why the generally accepted at the present time version of SRT would not be a success without it?

And the thing is that the corresponding to relativistic formulas of SRT - for example, the formula of Lorentz-Einstein

$$
m=\frac{m_{0}}{\sqrt{1-(v / c)^{2}}}
$$

where $m_{0}$ is the mass of the moving body rest (for example, an elementary particle);

$m$ is relativistic mass of the moving body;

$v$ is the speed of movement of the physical body;

$c$ is the speed of light;

- the physical values on the hyper light speeds when $v>c$ are taking imaginary values. And the authors of SRT did not know how to explain this result. So they were forced either to confess that they do not fully understand their theory, or in some way to avoid the need for such confession. Otherwise, SRT would look unfinished and inconclusive.

So in order to avoid such a situation the authors of SRT have needed proven anywhere and by anyone (in other words, postulated) principle of non-exceeding the speed of light, as in accordance with this principle at sub-light speeds when $v<c$ appropriate to relativistic formulas values received quite understandable and interesting for interpretation real values. And in hyper light speeds at speeds $v>c$, it would seem, it is no longer necessary to explain anything, since such a situation was supposedly impossible

And this postulate was substantiated at first glance even quite clearly as follows. Since the value of the relativistic mass $m$ in the formula (1) depends on the speed $v$, then at near-light speeds when approaching $v<c$ the mass $m$ takes values approximating infinitely large real values, i.e., $\lim _{v \rightarrow c-0} m(v)=+\infty$. But then to overcome the astrophysical point of singularity $v=c$ infinitely large energy becomes necessary. By virtue of what it proved impossible to overcome the light-speed barrier. And so, it was concluded that there is nothing behind this barrier, i.e., we live in the monoverse, i.e. in the only real universe.

But the provided substantiation is refuted even by practice of usual life. Indeed, it is impossibility to get into the next room of one's dwelling through the barrier separating them in the form of wall, does not mean that the next room does not exist and that it is impossible to get there through the door.

\section{The Proof of Principle of the Physical Reality of Imaginary Numbers}

It is interesting to note that the current version of SRT was actually refuted even before its creation. Indeed, the main works of the authors of SRT [1] [2] [3] [4], were published in 1897-2005, and in 1897 an engineer and inventor Charles Proteus Steinmetz has offered his interpretation of Ohm's law applied to the linear electric circuits of alternating current [18], which allowed to prove the physical 
reality of imaginary numbers the simplest and most convincing way. Thus, with its help the uselessness in SRT of erroneous principle of non-exceeding the speed of light could have been also proven.

But at that time the Internet had not yet existed, and it was excusable to the creators of SRT not know about Steinmetz's theory. But after a hundred plus years it is already unforgivable for the physicists not to know of established by Steinmetz theory, which is now is used by all electrical and radio engineers.

And not to be unfounded, let's give a simple proof of the physical reality of imaginary numbers [19] [20] [21] [22], which made the costly MINOS, OPERA and ICARUS experiments unnecessary. In contrast to discovered in $1826 \mathrm{Ohm}$ 's law, which was formulated for electric DC circuits, Steinmetz suggested the wording of this law for electric AC circuits. In accordance with the theory he proposed, not only resistors have the electrical resistance (or conductivity) whose resistance is measured by real numbers, but also capacitors and inductance coils, the resistance of which are measured by imaginary numbers with different polarities. Moreover, the value of imaginary resistance of capacitors and inductance coils depends on the frequency. Therefore, any resistance of any LCR-, RL-, RC- and LC-circuit is measured by complex numbers, the value of which also depends on the frequency applied to electrical voltage circuit.

This said, the resistance of capacitors and inductance coils measured by imaginary numbers is often also called imaginary. And under the dominant influence of SRT they are still understood as imaginary, that is, really physically not existing. But this is not true. If the resistance of the capacitors and inductance coils were actually imaginary, then from the change in their values when the change of frequency of the applied voltage the value flowing through LCR-, RL-, RC-, LC-circuit of the electric current would have been independent. But the engineers have known for a long time that it depends on the frequency. This is confirmed by the existence of mass-produced for several decades instruments for measuring the frequency characteristics of electrical circuits, which confirm the presence of the mentioned dependence.

Moreover, if the imaginary impedance of capacitors and inductance coils were not physically real, in electrical circuits could not be a resonance phenomenon [23] discovered in 1602 by Galileo di Vincento Bonaiutide' Galilei. And then there would be no such science as radio engineering, telecommunications, radar, television, and many others.

Consequently, since mathematics is the universal language of all exact sciences, the physical reality of imaginary resistance of capacitors and inductors clearly shows the physical reality of imaginary numbers which measure the parameters of corresponding physical objects. Therefore, the principle of the physical reality of imaginary numbers is general scientific and also applicable in special relativity theory, quantum mechanics, optics and the rest of the exact sciences.

There is other, equally compelling, evidence of the physical reality of imaginary numbers [13] [14] [15] [16] [17] [24] [25]. 


\section{Relativistic Formula of Revised Version of SRT, A Refutation of the Principle of Non-Exceeding the Speed of Light}

But from the principle of the physical reality of imaginary numbers, it follows that according to Lorentz-Einstein formula (1) relativistic mass $m$ at the hyper light speeds when $v>c$ as well as imaginary physical values corresponding to the rest of the relativistic formulas when $v>c$ are existing and physically real. And the principle of non-exceeding the speed of light turns out to be unnecessary. And then it becomes necessary to do what the creators of SRT were unable to do - to explain the meaning of these imaginary physical values [26].

This can be done as follows. Moving at the hyper light speeds $v>c$ are actually physically existing micro-objects with imaginary mass-for example, tachyons [27] [28] — cannot be registered by us (and we cannot see this kind of macro-objects) only because they are outside the event horizon. But because they still exist, they are not in our universe, but somewhere else. And this another place of their location will be called for certainty tachyon universe. And then let us call our universe tardyon universe.

And the explanation given above regarding physical nature of tachyons and tachyon universe actually is a refutation of the principle of non-exceeding the speed of light.

Therefore, we do not live in monoverse (i.e. in a single universe), as stated in the current version of SRT, but in the invisible Multiverse, which we shall call hidden [29]. And in its tachyon universe according to the first postulate of SRT, act the same as in our tardyon universe, physical, chemical and other laws of nature, it also has its own living inhabitants and its own super civilizations.

But Lorentz-Einstein formula (1) and other relativistic formula of the existing version of SRT do not comply with this condition, as in the ranges $v<c$ and $v>c$ the character of their changes differs. Therefore, the Lorentz-Einstein formula should be corrected as follows:

$$
m=\frac{m_{0}(i)^{q}}{\sqrt{1-(v / c-q)^{2}}}=\frac{m_{0}(i)^{q}}{\sqrt{1-(w / c)^{2}}}
$$

where $q=\lfloor v / c\rfloor$ is discrete function "floor" from the argument $v / c$;

$w=v-q c$ is its own for each local universe speed, which can only take values in the range $0 \leq w \leq c$;

$v$ is the speed, measured from our universe, which can therefore be called an total speed;

$c$-is the speed of light;

$i=\sqrt{-1}-$ is an imaginary unit.

The rest of the relativistic formula can be corrected in a similar manner. For example,

$$
\Delta t=\Delta t_{0}(i)^{q} \sqrt{1-(v / c-q)^{2}}=\Delta t_{0}(i)^{q} \sqrt{1-(w / c)^{2}}
$$


where $\Delta t_{0}$ is the rest time;

$\Delta t$ is relativistic time.

It should be noted that Albert Einstein did not exclude such SRT correction in the future. He wrote: "No single idea, which I would be sure that it will stand the test of time".

\section{Hidden Multiverse}

\subsection{The Structure of the Hidden Multiverse}

From the formulas (2a, b), as it is seen that the parameter $q=0$ corresponds to the tardyon universe (since $i^{0}=1$ ), and the parameter $q=1$ corresponds to the tachyon universe (since $i^{1}=i$ ), but it does not mean that in the hidden Multiverse there should be only two universes. It is proved later that in fact there are more than twenty of them in the hidden Multiverse. Then tardyon antiverse will correspond to the parameter $q=2$ (since $i^{2}=-1$ ), parameter $q=3$ will correspond to the tachyon antiverse (since $i^{3}=-i$ ), parameter $q=4$ will correspond to the other tardyon universe (since $i^{4}=1$ ), parameter $q=5$ will correspond to another tachyon universe (since $i^{5}=i$ ), etc.

It is possible that something similar Stephen William Hawking had in mind, who wrote: "Imaginary time is a new dimension, at right angles to ordinary, real time."

At the same time new parameter $q$ in the formulas (2a, b) can be interpreted in the hidden Multiverse as a fourth spatial coordinate (similar to apartments number in an apartment building, each of which contains its own three spatial coordinates). Consequently, parallel universes (because, despite its immensity, they never intersect) in such a structure of Multiverse co-exist in the fourdimensional space, forming a closed (otherwise the question arises what is beyond the edges of the open Multiverse structure) helically-formed ring, as shown in Figure 1.

And these parallel universes in multidimensional space, drifting continuously, sometimes touch each other (as the people in the crowd), and sometimes even partially immerse in each other, while forming a certain transition zones, through which parallel universes can share their material content.

These numerous transition zones between adjacent universes are often referred to in the fiction as portals or stellar gates [30], in which the value of the parameter $q$ changes smoothly (while in the parallel universes the value of the parameter $q$ is constant), are shown in Figure 1 by means of single bi-directional arrows.

\subsection{Explanation of the Dark Matter and Dark Energy}

However, any structure of the Multiverse cannot be considered satisfactory in any way, if it is does not explain the phenomenon of dark matter and dark energy [31] [32]. Not only astrophysical, but general scientific worldview importance of this problem is caused by the facts that: 


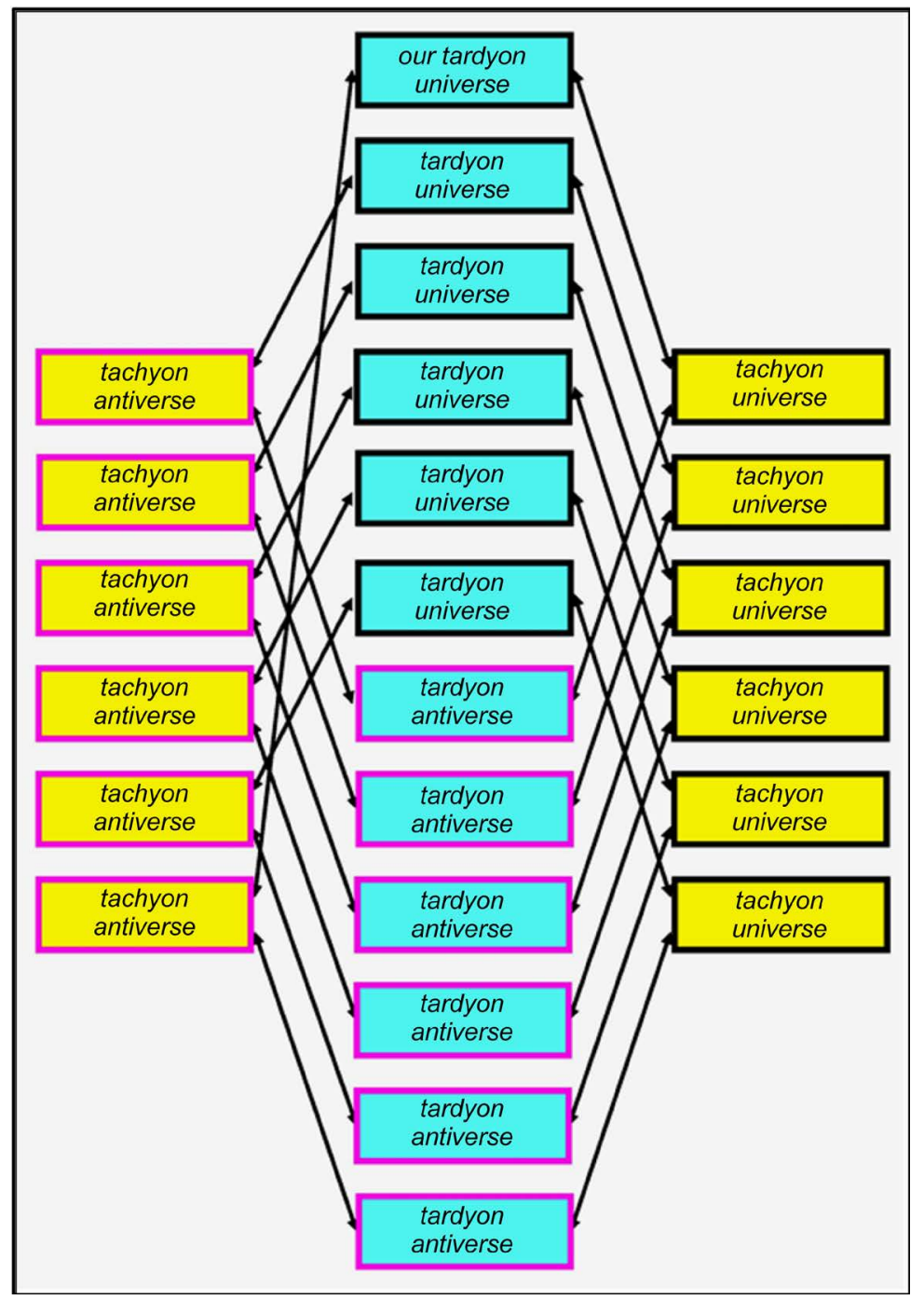

Figure 1. Possible structure of the hidden Multiverse corresponding to the physical reality of complex numbers.

1) mass-energy of the dark matter and dark energy exceeds the mass-energy of our entire visible universe more than twenty times;

2) in the composition of dark matter and dark energy none of the known chemical elements is found, and even any subatomic particle that gives rise to doubts as to the correctness of the modern understanding of the term "matter" and is expected to cast a science in its development up to millennia ago.

In this connection, Stephen William Hawking wrote: "The missing link in cosmology is the nature of dark matter and dark energy".

This phenomenon has not been explained up until very recently. Moreover, its explanation failed to even come close in the past decade. But the reason for that is just the wrong statement of the search task of explaining the phenomenon certainly in monoverse corresponding to the existing incorrect version of SRT. And as Albert Einstein claimed: "Insanity. doing the same thing over and over again and expecting different results".

But if we abandon this restrictive condition and will seek a solution to the 
problem in the hidden Multiverse, we won't even have to look for it. It is obvious [33]-[39]:

1) dark matter and dark energy are the invisible other, except our parallel universes of the hidden multiverse, i.e., these are only their other names;

2) at the same time the dark matter are the universes surrounding our universe;

3) dark energy are the others, except for our and neighboring with it universes, other universes;

4) and the chemical composition of the contents of the universes that make up the dark matter and dark energy, and being in other dimensions, cannot be determined from our universe.

\subsection{Explanation of Where the Antimatter Is Located}

No less important and misunderstood problem in cosmology than the phenomenon of the dark matter and dark energy is the explanation of antimatter location [40] [41].

Indeed, the Big Bang which occurred $13.77 \pm 0.059$ billion years ago had to create matter and antimatter in equal amounts. But our universe consists of the matter only. The scientists were able to synthesize antimatter, as well as to discover it in some natural phenomena only in minute amounts, which, however, confirms the fundamental possibility of its existence.

So the logical question is - where the antimatter is located in large quantities? Clearly, there's no place for it in the monoverse. Otherwise, as a result of the annihilation of matter and antimatter the monoverse would have been destroyed.

And in the hidden Multiverse as a result of corresponding in a multidimensional space alternation of universes and antiverses, both tardyon and tachyon, it can stably exist. Moreover, as shown in Figure 1, Figure 2 and Figure 4 it may have even more pairs of universes and antiverses [42].

\subsection{Quaternion Structure of the Hidden Multiverse}

Extremely valuable data obtained by the spacecraft WMAP [43], launched in 2001, and Planck [44], launched in 2009, allow to significantly clarify the structure of the hidden Multiverse.

According to the measurements of Planck [44] the whole universe (in fact, the whole hidden Multiverse) is made up of baryonic matter by 4, 9\% (and according to the previous measurements of WMAP - by $4.6 \%$ ), by $26.8 \%$ (according to WMAP measurements-by 22.4\%) of the dark matter, and by $68.3 \%$ (according to WMAP measurements-by $73.0 \%$ ) of dark energy.

On the basis of these data it is safe to assume that as a result of mutual infiltration through the portals of micro- and mini-content of the parallel universes, their mass-energy has the largely averaged out (even if, for some reason, immediately after the Bing Bang their mass-energy in different universes would have been different), and it is with great accuracy equal to the mass-energy of our universe. 


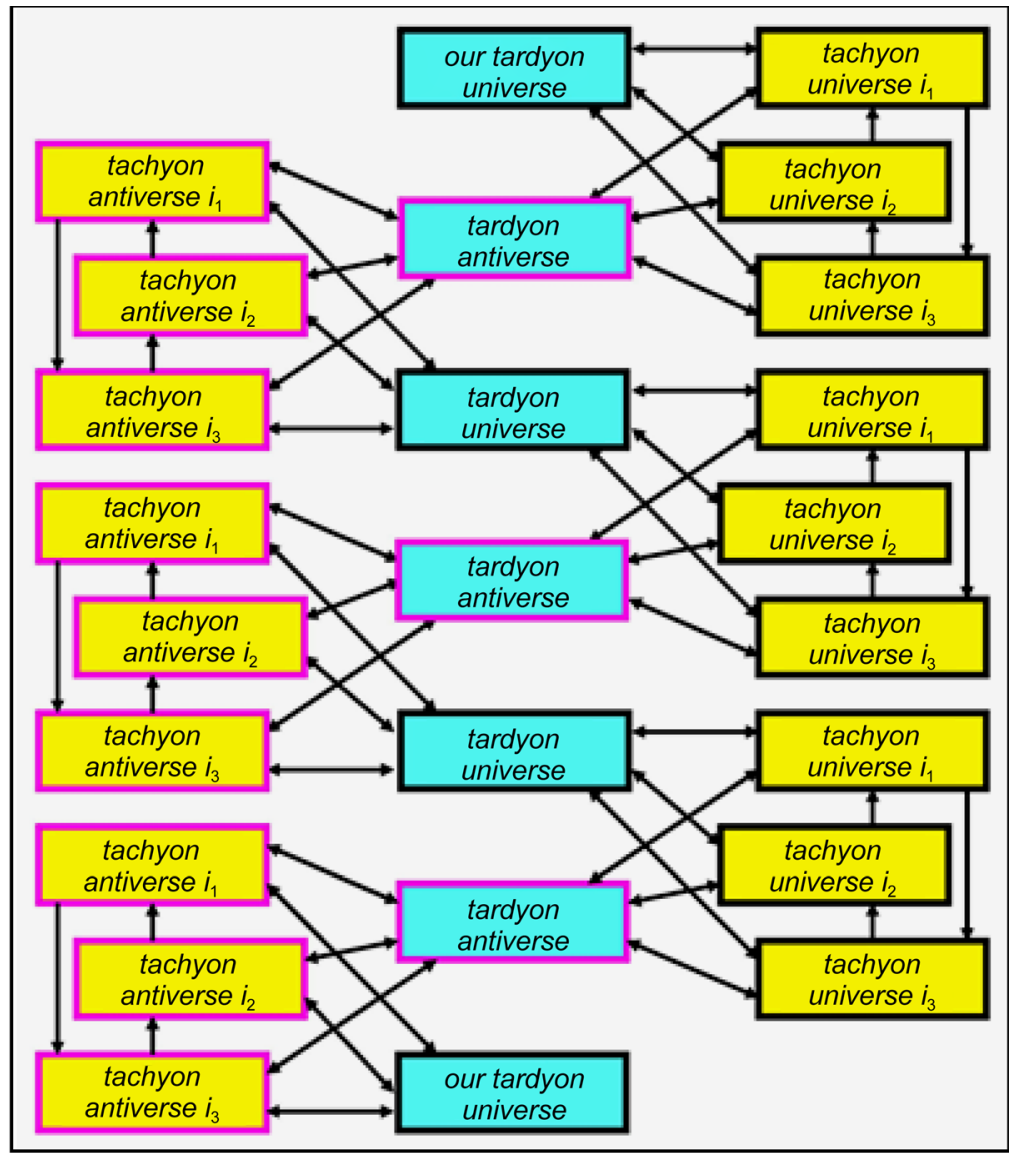

Figure 2. Possible structure of the Multiverse, corresponding to the physical reality of quaternions.

Therefore:

1) According to Planck data the hidden Multiverse contains $100 \% / 4.9 \%=20.4$ (and according to WMAP - $100 \% / 4.6 \%=21.8$ ) parallel universes. I.e. the most probably $20 \ldots 22$ parallel universes.

2) According to Planck data the hidden Multiverse contains $26.8 \% / 4.9=5.5$ (and according to WMAP $22.4 \% / 4.6 \%=4.9$ ) parallel universes. I.e. the most probably 5 ... 6 parallel universes.

3) According to Planck data the hidden Multiverse contains $68.3 \% / 4.9=13.9$ (and according to WMAP $73.0 \% / 4.6 \%=15.9$ ) parallel universes. I.e. the most probably $14 \ldots 16$ parallel universes.

However, it is not difficult to note that these results do not correspond to the structure of the hidden Multiverse, as shown in Figure 1. In fact, in the structure of the hidden Multiverse shown above our tardyon universe had only two neighboring universes, rather than 5 ... 6 universes. Consequently, some important facts when configuring this structure had not been taken into account.

And what kind of circumstances are those, which may have conditioned the emergence of $5 \ldots 6$ tachyon neighboring universes and antiverses in our tardyon universe? 
To answer this question, let us recall that the tachyon universes and antiverse in the above structure of the hidden Multiverse were a consequence of accounting in the formulas $(2 a, b)$ of the physical reality of only one imaginary unit, which is typical for complex numbers, and statement of the existence of only one additional spatial measurement. Therefore, in this situation it is logical to assume that for the existence of 5 ... 6 neighboring tachyon universes and antiverses requires three additional spatial dimensions and accounting for physical reality of hyper complex numbers [45], containing three imaginary units $i_{1}, i_{2}, i_{3}$. And these three imaginary units $i_{1}, i_{2}, i_{3}$ in quaternions are interconnected by the co-relations

$$
\begin{aligned}
& i_{1}^{2}=i_{2}^{2}=i_{3}^{2}=1 \\
& i_{1} i_{2} i_{3}=i_{2} i_{3} i_{1}=i_{3} i_{1} i_{2}=-1 \\
& i_{1} i_{3} i_{2}=i_{2} i_{1} i_{3}=i_{3} i_{2} i_{1}=1
\end{aligned}
$$

Consequently, Lorentz-Einstein formula should be corrected once more as follows

$$
m=\frac{m_{0}\left(i_{1}\right)^{q}\left(i_{2}\right)^{r}\left(i_{3}\right)^{s}}{\sqrt{1-[v / c-(q+r+s)]^{2}}}=\frac{m_{0}\left(i_{1}\right)^{q}\left(i_{2}\right)^{r}\left(i_{3}\right)^{s}}{\sqrt{1-(w / c)^{2}}}
$$

where $q$ is the total number of parallel universes, penetration to which with the distance from our tardyon universe is realized via portals corresponding to the imaginary unit $i_{1}$;

$r$ is the total number of parallel universes, penetration to which with the distance from our tardyon universe is realized via portals corresponding to the imaginary unit $i_{2}$;

$s$ is the total number of parallel universes, penetration to which with the distance from our tardyon universe is realized via portals corresponding to the imaginary unit $i_{3}$;

$w=v-(q+r+s) c-$ is its local to the respective universe speed, which can take values only in the range of $0 \leq w \leq c$;

$v$ is the speed, measured from our tardyon universe, that is why let's call it the total speed;

$c$ is the speed of light.

Other relativistic formula of SRT can be corrected in a similar manner. For example,

$$
\begin{aligned}
\Delta t & =\Delta t\left(i_{1}\right)^{q}\left(i_{2}\right)^{r}\left(i_{3}\right)^{s} \sqrt{1-[v / c-(q+r+s)]^{2}} \\
& =\Delta t\left(i_{1}\right)^{q}\left(i_{2}\right)^{r}\left(i_{3}\right)^{s} \sqrt{1-(w / c)^{2}}
\end{aligned}
$$

The quaternion structure of the hidden Multiverse [46] corresponding to the formulas $(4 a, b)$ in the form of a closed helical ring is shown in Figure 2. There the single bilateral arrows indicate numerous bidirectional portals corresponding to the ratio (3a), and the single unilateral arrows indicate numerous one-way portals, corresponding to the ratios (3b) and (3c). At the same time, our tardyon 
universe, corresponding in the closed helical ring of such structure of the hidden Multiverse to the relative beginning and end of the reference point of reference, is shown twice to simplify the drawing.

The structure of the six-dimensional space, in which such a hidden Multiverse exists, is illustrated in Figure 3.

However, given in Figure 2 structure of the hidden Multiverse is still not fully in line with the results of calculations given above, as it contains not $20 \ldots 22$ parallel universes but 24 universes. Therefore, some $2 \ldots 4$ parallel universes should be excluded out of it, as shown for example in Figure 4. However, the structure of the hidden multiverse thus obtained has edges, which give rise to the question-what is behind these edges? And the most natural response to this question would be a statement that there are other Multiverses behind them (possibly with a different structure-for example, in the form of an open helical ring similar to the closed helical ring shown in Figure 1) that do not fall under our observations not only by electromagnetic but also by gravitational manifestations. In other words, it can be affirmed that there is not only our hidden Multiverse, but also other Multiverses, which together form the Hyperverse.

\subsection{Verifiability of the Hidden Multiverse}

Currently a large number of various implementations of the Multiverses are offered. The most interesting of them are set forth in [47]-[54]. However, all of them are unverifiable, i.e. even in the distant future they can neither be experimentally confirmed nor refuted. And that is why so many scientists are skeptical to the idea of the Multiverse-why study what actually does not actually exist. According to them (and the author agrees with them) the efforts aimed at solving the real secrets of nature are much more important.

But the idea of the hidden Multiverse outlined above is verifiable [55], because:

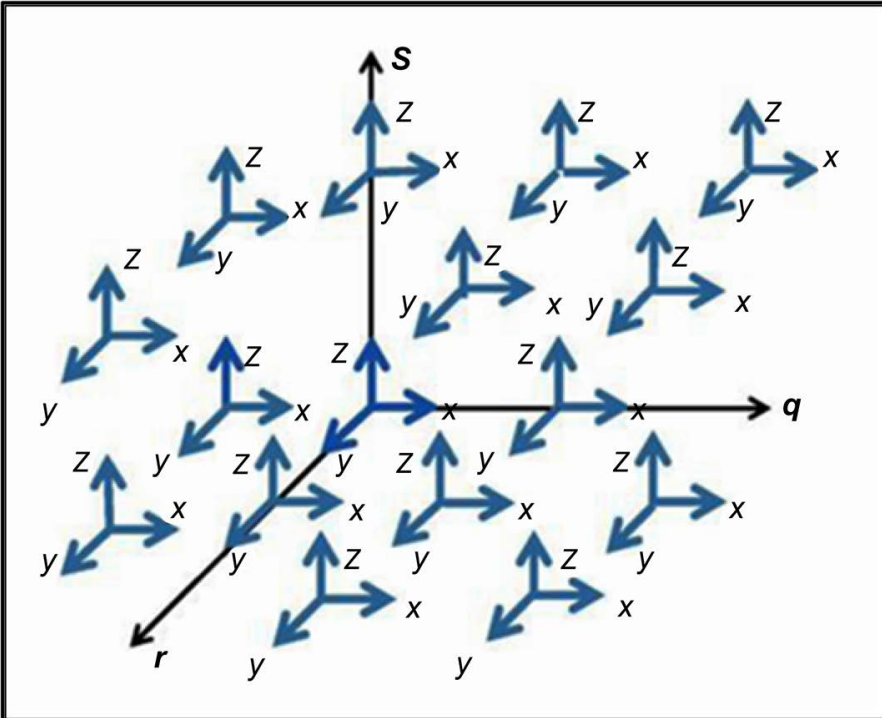

Figure 3. The six-dimensional space of the hidden Multiverse. 


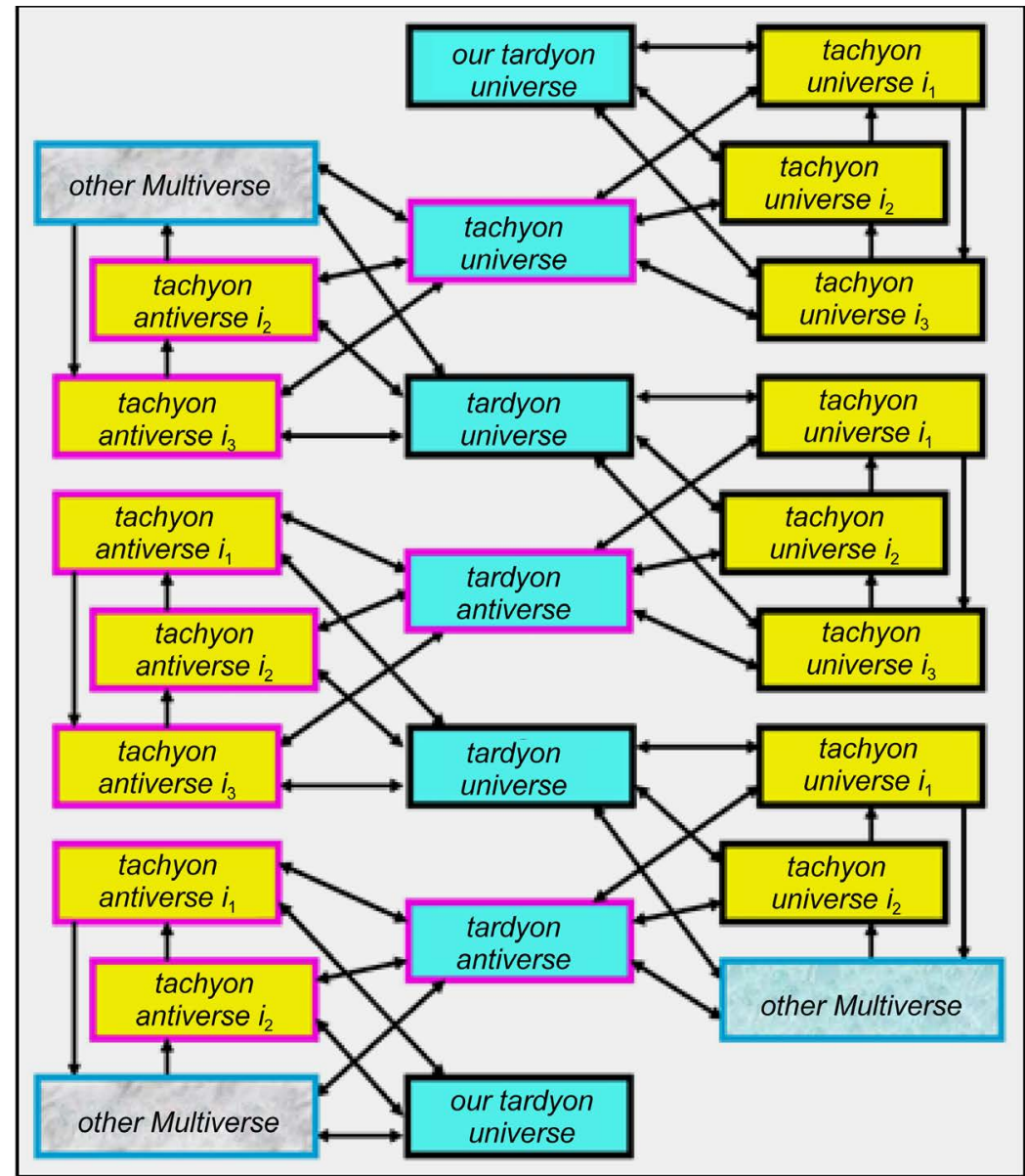

Figure 4. Hypothetic structure of the hidden Multiverse, corresponding to the physical reality of quaternions.

1) it has experimental support in the form of the dark matter and dark energy, as the latter are merely other names of all the others, except for our parallel universes forming it;

2) it has also a confirmation in the form of those experiments on particle accelerators, as a result of which there was a mass defect which was explained by the tachyons formation and passage through the microportals to the neighboring universes;

3) it can be experimentally confirmed by geophysical research of the portals, which are so-called anomalous zones at the Earth [56], and subsequent people visits of the neighboring universes (if their inhabitants will allow us to do it).

\section{The Problem of Contacts with the Inhabitants of the Hidden Multiverse}

Since it follows from the foregoing that the neighboring with our universe other parallel universes are actually physically exist and that the portals connecting our universe with them actually physically exist, including those located at the Earth, it is useful to consider the problem with contacts inevitably existing around us other civilizations as well [57]. 


\subsection{Types of Civilizations}

And to do this, to begin with, let's clarify the basic concepts on this issue.

Under civilization we will understand the hierarchical structure of living creatures, united for the common good and able to mutually coordinate their activities by exchanging the information necessary for it, i.e. having a language. And under super civilization we will understand a civilization more advanced than our human civilization.

Currently, it is widely believed that in the world there is only one civilization human. And that only people able to talk to each other. However, it is not like this.

Firstly, the humanity itself is not a single civilization, but is divided according to the territorial, economic, cultural, religious, linguistic, racial and historic grounds into sub civilizations. And these sub civilizations meet different levels of their development.

Secondly, according to the proposed definition civilizations are many biological communities as well-monkey and wolf packs, herds of elephants, and even the ant heels and bee swarms. But they differ significantly from human sub civilizations. And their main difference from the human sub civilizations is the lack of industrial production. Therefore, they, in contrast to human industrial sub civilizations, can be called non-industrial sub civilizations.

Finally, at the Earth is certainly present, a super civilization (or super civilizations) unknown to us as well, whose habitat extends beyond our planet, the solar system and even our universe. Therefore, this earthly super civilization is extraterrestrial as well. And it is unknown to us because it is well protected from human attention the same way as the people are protected from penetration in their homes of birds, animals, snakes, insects and other living inhabitants of the Earth.

And this super civilization perceives us nearly the same way as we perceive cat, dog and other non-industrial sub civilizations living next to us as intelligence tests, for example, we have not passed in the form of crop circles.

In the order to further explain how you can improve the human intellect, it is necessary to clarify this concept as well, by which we will mean the individual and collective ability to detect the patterns in the initial data array (in particular, to read encrypted messages) and to use identified patterns.

\subsection{The Problem of Contact with the Earth's Super Civilization}

The scientists have been unsuccessfully trying to solve the problem of contacts with extraterrestrial civilizations that exist in outer space since 1959 within the framework of SETI project (Search for Extraterrestrial Intelligence), which from 1971 is carried out under the auspices of NASA. And in 2015, Professor Stephen William Hawking, and billionaire Yuri Milner announced that for Breakthrough Listen initiative for the same purpose they have allocated another $\$ 100$ million.

However, the formulation of the problem to find other civilizations in outer space is still obviously had meaning only in so far as part of Monoverse hypo- 
thesis corresponding to the current version of SRT, it had no alternative. But, as shown above, the existing version of SRT was wrong. Therefore, the discovery of the existing in reality the hidden Multiverse made redundant search for extraterrestrial civilizations in outer space. Such searches are meaningless because you can simply walk through numerous, located at the territory of almost all countries, portals, called anomalous zones to the neighboring universes [56]. And it is clear that sooner or later the inhabitants of all six neighboring universes realized this. And the most advanced and powerful of these neighboring civilization established control over the portals at the Earth. This most advanced civilization has found us long time ago. And it has on our planet for a long time.

As for the anomalous zones, they are called in such a way because something strange is happening there, which is why people are afraid to visit these places. And rightly so, as the inhabitants of other universes do not want people to visit them (because we also do not want uninvited guests to visit our homes), that is why they place in the portals the different spooks. And in addition to spooks the portals, probably are additionally equipped with security systems protecting the inhabitants of the neighboring universes from curiosity of the most intrepid inhabitants of the Earth. All this must be taken into account.

And although it would seem that research of the portals with the help of robots may seem as a safe pastime and even bringing great benefit, since, after realizing the geophysical processes in the portals, you can create artificial portals, including time machines (as the time in different universes flows in different directions), it should not be done so without the permission of super civilizations.

Therefore, the problem for us is not in the search of super civilizations, and not even not in search of contact with them, because to be or not to be of our contacts with super civilizations-it is not us who decide, but they are. Therefore, such contacts could take place only if our human civilization will be of interest to the Earth's super civilization. To do this, obviously, human civilization must be harmless for them, useful, and have a sufficiently high intelligence. Therefore, we still have to pass the test in the form of crop circles.

And in order to better understand the problem of contacts, it would be useful for us to learn how to establish meaningful contacts with terrestrial non-industrialized sub civilizations-communities of monkeys, dogs, cats, elephants, dolphins, etc.- - and understand them, since they may have knowledge unknown to us. And yet, because we do not know how the highly intelligent inhabitants of other universes look like.

\subsection{Creation of Human Super Intelligence}

That is why now the main task of mankind in space is not the search for extraterrestrial super civilizations and not attempts to establish contact with them, but much more difficult problem of self-perfecting of our human civilization to a level of extraterrestrial super civilizations.

The political component of this problem should to be addressed by politicians and diplomats. Economic components should to be addressed by businessmen 
and politicians again. A scientific component of this problem, which we would consider further, is, first of all, to find ways of creating human super intelligence. It should be solved by scientists and engineers specializing in the field of information technology.

To understand how this problem can be solved, first we need to clarify what is human intelligence, that is artificial intelligence and what is super intelligence. And also to remember what is technological singularity.

And let's start with technological singularity by which the point of time is understood - according to various estimates within 2018 or at the latest 2030 or at the latest 2045 year-after which as a result of Moore's law computers and the Internet allegedly will become so sophisticated that there will be computer civilization at the Earth (or computer super intelligence) that can enslave people. But this is-just an interesting fiction of talented writers of science fiction, which made a great impression on readers, including some scientists and engineers [58] [59].

In fact, the concept of technological singularity is unrealistic, since it ignores the fact that humanity is already using extensively a large number of dangerous technologies (for example, experiments to create artificial black holes at the Large Hadron Collider), over which they learned to maintain reliable control. And inevitably as a result of scientific and technological progress more and more of these dangerous technologies will be used. And, therefore, there also will be more and more opportunities for inventing horror stories.

But this is just another reason for more serious attitude to the problems of ozone depletion, global ocean pollution, increasing of carbon dioxide concentrations in the atmosphere and other adverse consequences of unwise human activities. But it is not an excuse to harass with mythical dangers and diversion of resources on unnecessary activities.

The weak point of technological singularity concept is the assertion that it is possible to create super intelligence on the basis of artificial intelligence and the Internet. In [60] [61] [62] it explains that this assertion is erroneous, since it does not take into account:

1) neither degree of perfection of the human intelligence, which uses a different set of very useful (but poorly studied) forms of human thought, including the powerful subconscious multivariate mind used, in particular, at the decision of creative tasks;

2) neither degree of imperfection of artificial intelligence, which is only poorly mimics the most primitive form of human thought-small-factor rational thinking;

3) nor degree of imperfection of the Internet, which has many fatal flaws, making it virtually unusable (except for e-mail and the World Wide Web WWW) in business, research and others kinds of people activity.

But on the basis of multivariate thinking the human super intelligence, capable of solving creative problems in science, in business, in the administration management and other kinds of human activity can be realized. For the imple- 
mentation of the human super intelligence the existing computers need other software, it is quite feasible, and special network support, for which, however, the Internet is fundamentally not capable. Using of computer networks [63] [64] [65] [66] for the same purpose, which instead of the packet communication will use noiseless coding will allow to solve such problems successfully and at the same time will make the computer super intelligence guaranteedly infeasible.

Finally, the danger of technological singularity is most incontrovertibly refuted by the fact of the existence of super civilizations themselves.

The human civilization, having learned new information super intellectual technologies and having overcome computer spooks, would already be able to establish contacts with super civilizations

\section{Conclusions}

The article shows that:

1) The principle of non-exceeding the speed of light was postulated in SRT (as it is not proven anywhere, and by anyone) only because its creators did not know how to explain the physical meaning of the imaginary relativistic mass, imaginary relativistic time and other imaginary relativistic physical values at hyper light speeds;

2) The principle of non-exceeding the speed of light is wrong;

3) Out of incorrect principle of non-exceeding the speed of light in SRT wrong conclusions about the existence of Monoverse and about physical unreality of imaginary numbers were made;

4) Imaginary, complex and hypercomplex numbers are physically real;

5) Relativistic formulas of SRT are incorrect and they are corrected;

6) The corrected relativistic formulas of SRT imply the existence of mutually invisible parallel universes (because, despite their immensity, they never intersect), together forming a hidden Multiverse;

7) The hidden Multiverse according to WMAP and Planck data has quaternion structure;

8) The hidden Multiverse according to WMAP and Planck data is combined with $1 \ldots 3$ other Multiverses, inaccessible to our observations, not only by electromagnetic but also by gravitational manifestations, which together form Hyperverse;

9) The hidden Multiverse according to WMAP and Planck data contains $20 \ldots$ 22 parallel universes, out of which 5 ... 6 parallel universes are neighboring to our universe, and the remaining $14 \ldots 16$ parallel universes are shielded from us by adjacent parallel universes;

10) Other parallel universes neighboring to our universe, form the dark matter and they are merely their other name;

11) Parallel universes, shielded from our universe with parallel universes of the dark matter, form dark energy and are merely their other name;

12) The dark matter and dark energy are the experimental evidence of physical reality of the hidden Multiverse; 
13) The possibility of the existence in the hidden Multiverse of a large number of anti-verses is determined by their alternation with the universe in six-dimensional space, due to which their annihilation is excluded;

14) Verifiability of the hidden Multiverse, in addition to the phenomenon of the dark matter and dark energy, is also confirmed by the experiments on particle accelerators, as a result of which there was a mass defect explained by the formation of tachyons and their transition into tachyon universes and antiverses;

15) Parallel universes drifting in a multidimensional space sometimes or always touch each other and even partially sink into each other, forming numerous transition zones, called portals or anomalous zones through which mutual transition of micro and mini objects-from subatomic particles to the living inhabitants with their vehicles is possible;

16) Search of extraterrestrial civilizations in outer space and attempts to establish contact with them are meaningless, because these civilizations have found us themselves long time ago, they have been presented at the Earth for a long time, and they, but not we decide whether there would be or would not to be such contacts;

17) To ensure that our contacts with the more advanced civilizations took place, human civilization must not pose a threat to them, should be useful for them and should significantly improve its intellectual level;

18) To implement the human multifactorial super intelligence, unlike the computer artificial intelligence which is a poor imitation of the most primitive type of human thought-small-factor rational thinking, the network support is necessary, for which the Internet is not suitable.

As you can see, this article contains some unusual statements. But on this occasion is appropriate to recall the opinion of Sir Isaac Newton: "No great discovery was ever made without a bold guess."

\section{Acknowledgements}

The author is grateful for participation in the discussion of the paper to Dr. Olga Ilyinichna Antonova, whose criticism and valuable comments contributed to improvement of the paper.

\section{References}

[1] Larmor, J.J. (1897) A Dynamical Theory of the Electric and Luminiferous Medium. Part III. Relations with Material Media. Philosophical Transactions of the Royal Society A: Mathematical, Physical and Engineering Sciences, 190, 205-300. https://doi.org/10.1098/rsta.1897.0020

[2] Lorentz, H.A. (1899) Simplified Theory of Electrical and Optical Phenomena in Moving Systems. Proceeding of the Royal Netherlands Academy of Arts and Science, 1, 427-442.

[3] Poincaré, H. (1905) On the Dynamics of the Electron. Comptes Rendus, 140, 15041508.

[4] Einstein, A. (1905) Zur Elektrodynamik bewegter Korper. Annalen der Physik, 17, 891-921. https://doi.org/10.1002/andp.19053221004 
[5] Einstein, A. (1920) Relativity: The Special and General Theory. H. Holt and Company, NY.

[6] Bohm, D. (2006) The Special Theory of Relativity, Routledge. Abingdon-onThames.

[7] Hawking, S.W. and Penrose, R. (2010) The Nature of Space and Time. Princeton University Press, Princeton. https://doi.org/10.1515/9781400834747

[8] Antonov, A.A. (2014) Verification of the Second Postulate of the Special Relativity Theory. Global Journal of Science Frontier Research: A Physics \& Space Science, 14, 51-59. https://doi.org/10.17686/sced_rusnauka_2014-901

[9] Adamson, P., Ashby, N. and Bumgarner, R. (2007) Measurement of the Velocity of the Neutrino with MINOS. arXiv:1408.6267v1 [physics.acc-ph]

[10] Adam, T., Agafonova, N., Aleksandrov, A., et al. (2011) Measurement of the Neutrino Velocity with the OPERA Detector in the CNGS Beam. arXiv:1109.4897v4 [hep-ex]

[11] Antonello, M., Baibussinov, B., Boffelli, F., et al. (2012) Precision Measurement of the Neutrino Velocity with the ICARUS Detector in the CNGS Beam.

[12] Popper, K.R. (2002) Conjectures and Refutations. The Growth of Scientific Knowledge. Routledge, London.

[13] Antonov, A.A. and Bazhev, V.M. (1970) Means of Rising Deflecting Currents for Spiral Beam Sweep on the CRT Screen. USSR Patent No 433650.

[14] Antonov, A.A. (2009) Resonance on Real and Complex Frequencies. European Journal of Scientific Research, 28, 193-204.

https://doi.org/10.17686/sced_rusnauka_2009-885

[15] Antonov, A.A. (2010) Solution of Algebraic Quadratic Equations Taking into Account Transitional Processes in Oscillation Systems. General Mathematics Notes, 1 , 11-16. https://doi.org/10.17686/sced_rusnauka_2010-887

[16] Antonov, A.A. (2010) New Interpretation of Resonance. International Journal of Pure and Applied Sciences and Technology, 1, 1-12. https://doi.org/10.17686/sced_rusnauka_2010-888

[17] Antonov, A.A. (2010) Oscillation Processes as a Tool of Physics Cognition. American Journal of Scientific and Industrial Research, 1, 342-349. https://doi.org/10.5251/ajsir.2010.1.2.342.349

[18] Steinmetz, C.P. (2010) Theory and Calculation of Electric Circuit. Nabu Press, Charlstone.

[19] Antonov, A.A. (2015) Adjustment of the Special Theory of Relativity According to the Ohm's Law. American Journal of Electrical and Electronics Engineering, 3, 124 129.

[20] Antonov, A.A. (2015) Ohm's Law Explains Astrophysical Phenomenon of Dark Matter and Dark Energy. Global Journal of Physics, 2, 145-149.

[21] Antonov, A.A. (2016) Ohm's Law Is the General Law of Exact Sciences. PONTE, 72, 131-142. https://doi.org/10.21506/j.ponte.2016.7.9

[22] Antonov, A.A. (2016) Ohm's Law Refutes Current Version of the Special Theory of Relativity. Journal of Modern Physics, 7, 2299-2313. https://doi.org/10.4236/jmp.2016.716198

[23] Frova, A. and Marenzana, M. (2006) Thus Spoke Galileo: The Great Scientist's Ideas and Their Relevance to the Present Day. Oxford University Press, New York.

[24] Antonov, A.A. (2015) Physical Reality of Complex Numbers Is Proved by Research of Resonance. General Mathematics Notes, 31, 34-53. 
[25] Antonov, A.A. (2016) Physical Reality and Nature of Imaginary, Complex and Hypercomplex Numbers. General Mathematics Notes, 35, 40-63.

[26] Antonov, A.A. (2014) Correction of the Special Theory of Relativity: Physical Reality and Nature of Imaginary and Complex Numbers. American Journal of Scientific and Industrial Research, 5, 40-52.

[27] Tanaka, S. (1960) Theory of Matter with Superlight Velocity. Progress of Theoretical Physics, 24, 171-200. https://doi.org/10.1143/PTP.24.171

[28] Feinberg, G. (1967) Possibility of Faster-than-Light Particles. Physical Review, 155, 1089-1105. https://doi.org/10.1103/physrev.159.1089

[29] Antonov, A.A. (2015) Hidden Multiverse. International Journal of Advanced Research in Physical Science, 2, 25-32.

https://doi.org/10.17686/sced_rusnauka_2015-903

[30] Antonov, A.A. (2016) Stargate of the Hidden Multiverse. Philosophy and Cosmology, 6, 11-27.

[31] Freeman, K. and McNamara, G. (2006) In Search of Dark Matter. Springer, New York.

[32] Nicolson, I. (2007) Dark Side of the Universe: Dark Matter, Dark Energy, and the Fate of the Cosmos. Johns Hopkins University Press, Baltimore.

[33] Antonov, A.A. (2015) Hidden Multiverse: Explanation of Dark Matter and Dark Energy Phenomena. International Journal of Physics, 3, 84-87.

https://doi.org/10.17686/sced_rusnauka_2015-903

[34] Antonov, A.A. (2015) Explanation of Dark Matter and Dark Energy Phenomena. Global Journal of Science Frontier Research: A Physics and Space Science, 15, 3338. https://doi.org/10.17686/sced_rusnauka_2015-902

[35] Antonov, A.A. (2015) Hidden Multiverse: Explanation of Dark Matter and Dark Energy Phenomena. Cosmology, 19, 40-61.

https://doi.org/10.17686/sced_rusnauka_2015-903

[36] Antonov, A.A. (2015) Why Dark Matter and Dark Energy Are Invisible? Optics, 4, 43-47.

[37] Antonov, A.A. (2015) The Astrophysical Phenomenon of Dark Matter and Dark Energy Proves the Existence of the Hidden Multiverse. American Journal of Modern Physics, 4, 180-188. https://doi.org/10.11648/j.ajmp.20150404.14

[38] Antonov, A.A. (2016) Explaining the Phenomenon of Dark Matter and Dark Energy by Existence of the Hidden Multiverse. Frontiers of Astronomy, Astrophysics and Cosmology, 2, 1-9.

[39] Antonov, A.A. (2016) Hypothesis of the Hidden Multiverse: Explains Dark Matter and Dark Energy. Journal of Modern Physics, 7, 1228-1246.

https://doi.org/10.4236/jmp.2016.710111

[40] Alfvén, H. (1966) Worlds-Antiworlds: Antimatter in Cosmology. W. H. Freeman \& Co., San Francisco.

[41] Frazer, G. (2004) Antimatter: The Ultimate Mirror. Cambridge University Press, Cambridge.

[42] Antonov, A.A. (2016) Dark Matter, Dark Energy and Antimatter Are Located in the Hidden Multiverse. PONTE, 72, 288-300. https://doi.org/10.21506/j.ponte.2016.9.22

[43] Hinshaw, G., Larson, D., Komatsu, E., et al. (2013) Nine Year Wilkinson Anisotropy Probe (WMAP) Observations: Cosmological Parameter Results.

[44] Adam, R., Ade, P.A.R., Aghanim, N., et al. (2015) Plank 2015 Results. 1. Overview of Products and Scientific Results.

[45] Korn, G.A. and Korn, T.M. (2000) Mathematical Handbook for Scientists and En- 
gineers: Definitions, Theorems, and Formulas for Reference and Review. Courier Dover Publications, New York.

[46] Antonov, A.A. (2015) Quaternion Structure of the Hidden Multiverse: Explanation of Dark Matter and Dark Energy. Global Journal of Science Frontier Research: $A$ Physics and Space Science, 15, 8-15.

[47] Deutch, D. (1998) The Fabric of Reality: The Science of Parallel Universes and Its Implications. Penguin Books, New York.

[48] Greene, B. (2000) The Elegant Universe: Superstrings, Hidden Dimensions, and the Quest for the Ultimate Theory. Random House Inc., New York.

[49] Steinhardt, P.J. and Turok, N. (2007) Endless Universe: Beyond the Big Bang. Doubleday, New York.

[50] Vilenkin, A. (2006) Many Worlds in One: The Search for Other Universes. Hill and Wong, New York.

[51] Carr, B. (2009) Universe or Multiverse? Cambridge University Press, Cambridge.

[52] Greene, B. (2011) The Hidden Reality: Parallel Universes and the Deep Laws of the Cosmos. Random House Inc., New York.

[53] Deutsch, D. (2012) The Beginning of Infinity: Explanations That Transform the World. Reprint Edition, Penguin Books, New York.

[54] Tegmark, M. (2015) Our Mathematical Universe: My Quest for the Ultimate Nature of Reality. Vintage, New York.

[55] Antonov, A.A. (2016) Verifiable Multiverse. Global Journal of Science Frontier Research: A Physics and Space Science, 16, 4-12.

[56] Chernobrov, V.A. (2000) Encyclopedia of mysterious places of the Earth. Publishing house "Veche", Moscow.

[57] Antonov, A.A. (2015) Where to Look for Alien Civilisations. Cosmology. Commentaries: Stephen Hawking's Aliens. The Search for Intelligent Extraterrestrial Life. Project Breakthrough Listen.

[58] Kurzweil, R. (2005) The Singularity Is Near: When Humans Transcend Biology. Viking, New York.

[59] Yudkowsky, E.S. (2006) Artificial Intelligence as a Positive and Negative Factor in Global Risk. Singularity Institute for Artificial Intelligence, Palo Alta, 1-41.

[60] Antonov, A.A. (2010) Human-Computer Super-Intelligence. American Journal of Scientific and Industrial Research, 1, 96-104. https://doi.org/10.5251/ajsir.2010.1.2.96.104

[61] Antonov, A.A. (2011) Human Super-Intelligence. International Journal of Emerging Sciences, 1, 164-173.

[62] Antonov, A.A. (2011) From Artificial Intelligence to Human Super-Intelligence. International Journal of Computer Information Systems, 2, 1-6.

[63] Antonov, A.A. (2009) Safe Global/Regional Informational Network. European Journal of Scientific Research, 28, 165-174.

[64] Antonov, A.A. (2012) The New Global Information Network Free from the Drawbacks of the Internet. ARPN Journal of Science and Technology, 2, 957-962.

[65] Antonov, A.A. (2013) New Global Computer Network. International Journal of Management, IT and Engineering, 3, 11-22.

[66] Antonov, A.A. (2013) New Business-Oriented Global/Regional Information Network. International Journal of Business Information Systems, 12, 321-334. https://doi.org/10.1504/IJBIS.2013.052453 
Submit or recommend next manuscript to SCIRP and we will provide best service for you:

Accepting pre-submission inquiries through Email, Facebook, LinkedIn, Twitter, etc. A wide selection of journals (inclusive of 9 subjects, more than 200 journals)

Providing 24-hour high-quality service

User-friendly online submission system

Fair and swift peer-review system

Efficient typesetting and proofreading procedure

Display of the result of downloads and visits, as well as the number of cited articles Maximum dissemination of your research work

Submit your manuscript at: http://papersubmission.scirp.org/

Or contact ns@scirp.org 